\title{
Tratamento intracístico de craniofaringioma com interferon alfa: relato de caso
}

\section{Intracystic Treatment of Craniopharyngiomas with Interferon Alpha: A Case Report}

\author{
${ }^{1}$ Médico Residente, Serviço de Neurocirurgia, Hospital e Maternidade \\ Celso Pierro, Pontifícia Universidade Católica (PUC), Campinas, SP, \\ Brasil; estágio em Neurocirurgia Oncológica no Hospital de Câncer \\ de Barretos, Barretos, São Paulo, Brasil \\ ${ }^{2}$ Médico Neurocirurgião, Serviço de Neurocirurgia Oncológica, \\ Hospital de Câncer de Barretos, Barretos, São Paulo, Brasil \\ 3 Fellowship em Neurocirurgia Oncológica, Serviço de Neurocirurgia \\ Oncológica, Hospital de Câncer de Barretos, Barretos, São Paulo, Brasil \\ ${ }^{4}$ Médica Pediatra, Serviço de Oncologia Pediátrica, Hospital de \\ Câncer de Barretos, Barretos, São Paulo, Brasil \\ ${ }^{5}$ Médico-Chefe, Serviço de Neurocirurgia Oncológica, Hospital de \\ Câncer de Barretos, Barretos, São Paulo, Brasil
}

Juliano Nery Navarro ${ }^{1}$ Rodrigo de Almeida Simon Sola ${ }^{1}$ Giusepe Picone Junior ${ }^{2}$ Carlos Roberto de Almeida Junior ${ }^{2}$ Ismael Augusto Silva Lombardi ${ }^{2}$ Allan Dias Polverini ${ }^{2}$ Guilherme Fonseca Bortoluzzi $^{3}$ Erica Boldrini ${ }^{4}$ Carlos Afonso Clara ${ }^{5}$

Address for correspondence Juliano Nery Navarro, MD, Rua Morgado de Mateus, $n^{\circ}$ 314, apto 31. São Paulo, SP, Brasil, CEP 04015-050 (e-mail: navarrojuliano@gmail.com).

Arq Bras Neurocir 2015;34:170-173.

\begin{abstract}
Resumo
Palavras-Chave

- interferon alfa

- craniofaringioma

- tratamento intracístico

Craniofaringiomas são tumores histologicamante benignos, extrínsecos ao parênquima cerebral. Visando ao controle da doença, preconiza-se, como tratamento padrão, a tentativa de ressecção completa, meticulosamente planejada com vistas a preservar as funções habituais do paciente. Outras formas de terapias podem ser utilizadas como alternativas ou como adjuvantes à cirurgia. Considerando-se que muitos craniofaringiomas apresentam componente cístico, a utilização de cateteres posicionados no interior do lúmen cístico para aplicação de agentes quimioterapêuticos, como o interferon alfa (IFNa), tem-se mostrado promissora. Administração intratumoral de 3 milhões de unidades de IFNa em dias alternados, totalizando doze ciclos, tem sido utilizada empiricamente. Relatamos o caso de uma paciente de 12 anos de idade, com diagnóstico de craniofaringioma de predomínio cístico, tratada no Hospital de Câncer de Barretos (HCB) utilizando a aplicação de IFNa semanalmente, que apresentou evolução favorável.
\end{abstract}

Craniopharyngiomas are histologicaly benign tumors, whose ideal brain treatment requires its complete surgical resection, while preserving most of the usual functions of the patient. Due to the complexity of the surgical procedure, related to location (surrounded by paraselar structures), it is often difficult to achieve its complete resection. Thus, alternative therapies have been reported, including the use of intracystic IFNa on alternate days. We report the case of a 12 year-old female, diagnosed
Recebido

May 27, 2014

Aprovado

March 31, 2015
DOI http://dx.doi.org/ $10.1055 / \mathrm{s}-0035-1554044$. ISSN 0103-5355.
Copyright $\odot 2015$ by Thieme Publicações License terms Ltda, Rio de Janeiro, Brazil $(\circledast) \Theta \circledast$ 
with cystic craniopharyngioma, weekly treated with intracystic IFNa, for a total of 12 cycles, with favorable outcome.

\section{Introdução}

Craniofaringiomas são tumores histologicamente benignos da região selar e suprasselar, ${ }^{1}$ extrínsecos ao parênquima cerebral, ${ }^{2}$ que correspondem de 1,2 a $4 \%$ dos tumores intracranianos pediátricos. ${ }^{3,4}$ Como tratamento ideal, visando ao controle da doença, preconiza-se a tentativa de ressecção completa, ${ }^{1,5}$ que deve ser meticulosamente planejada com vistas a preservar as funções habituais do paciente.

Na infância, tumores relacionados ao eixo hipotálamohipofisário afetam não somente o desenvolvimento neurocognitivo, mas também o desenvolvimento corporal-por meio de efeitos deletérios sobre os sistemas visual, endocrinológico e metabólico -, de modo mais significativo do que na maturidade. ${ }^{5,6}$ Devido à proximidade anatômica e ao envolvimento secundário e variável do aparato óptico, das artérias carótidas e cerebrais anteriores, da hipófise e do hipotálamo, sua localização estabelece como desafio técnico a ressecção cirúrgica completa. ${ }^{4,6,7}$ Nas crianças, até $90 \%$ dos craniofaringiomas apresentam componente cístico ${ }^{5,8,9}$, em cujo interior podem ser posicionados cateteres para aplicação de agentes quimioterápicos capazes de contraí-lo (como o IFNa) - estratégia que se tem mostrado minimamente invasiva e promissora. ${ }^{1,2,5,7-11}$

O interferon alfa (IFNa) é uma glicoproteína pertencente à família das citocinas, cujas propriedades antitumorais se devem à ação antiproliferativa, citotóxica e imunomoduladora. ${ }^{8}$ Sua eficácia antitumoral, por indução de apoptose, já está bem estabelecida em relação ao carcinoma de células escamosas de pele. ${ }^{12}$ Jakacki et al. ${ }^{13}$ foram pioneiros na utilização sistêmica de IFNa para tratamento de craniofaringiomas recorrentes ou refratários. Posteriormente, baseandose em suas experiências com bleomicina, ${ }^{14}$ Cavalheiro et al. ${ }^{8,10}$ propuseram, empiricamente, a administração intracística de 3 milhões de unidades de IFNa de 3 a 4 vezes por semana.

O seguinte relato apresenta nossa experiência com a utilização de IFNa administrado via cateter de Ommaya por meio de posologia ainda não descrita até o momento.

\section{Relato de Caso}

A.L.P.C., de 12 anos de idade, sexo feminino, natural e procedente de Uberlândia, Minas Gerais, foi encaminhada ao serviço de neurocirurgia do Hospital de Câncer de Barretos para a segunda avaliação, com diagnóstico de craniofaringioma, considerado inoperável, em agosto de 2012.

Em 2005, a paciente começou a apresentar cefaleia persistente. A investigação diagnóstica destacou presença de hipopituitarismo, e exames de imagem evidenciaram lesão suprasselar predominantemente cística associada a hidrocefalia obstrutiva. A paciente fora submetida a procedimento cirúrgico para drenagem do conteúdo cístico, instalação de sistema de derivação ventriculoperitoneal e ressecção do componente sólido, com melhora subsequente dos sintomas. O diagnóstico anatomopatológico revelou tratar-se de craniofaringioma adamantinomatoso grau I da Organização Mundial da Saúde. Seis anos após essa abordagem, a paciente apresentou novos episódios de cefaleia persistente. Exames de ressonância nuclear magnética mostraram recidiva do conteúdo cístico e discreto aumento do componente sólido, sendo realizado novo procedimento cirúrgico com vistas à drenagem do cisto e ressecção do componente sólido parcialmente obtida. Como o remanescente continuava a exibir crescimento lento e progressivo, acompanhado de deterioração do estado geral, a paciente foi encaminhada para nosso serviço.

Admitida com cefaleia, sonolência e labilidade emocional, foi submetida à realização de exames de imagens que evidenciaram presença de lesão expansiva predominantemente cística suprasselar, compatível com recidiva de craniofaringioma (-Fig. 1). Procedemos à drenagem parcial do componente cístico e instalação por via endoscópica de cateter com reservatório subcutâneo de Ommaya, bilateralmente, para aplicação de IFNa. Após o procedimento, a paciente apresentou melhora clínica significativa e reversão do quadro neurológico. A tomografia computadorizada de crânio pós-operatória para verificação de posicionamento dos cateteres intracísticos mostrou cateter direito em situação adequada (-Fig. 2) e cateter esquerdo em localização insatisfatória para realização do procedimento (dado que sua extremidade perfurava a parede posterior do cisto)(-Fig. 3 ). Foi proposta inicialmente a aplicação intracística de IFNa, 3 milhões de unidades, em dias alternados, conforme descrito por Dastoli e Cavalheiro et al., 8,10 associado a aspiração do conteúdo cístico, até totalizar doze ciclos, pelo cateter direito. Entretanto, devido à impossibilidade dos familiares seguirem esse protocolo (dificuldades trabalhistas, distância do hospital em relação ao domicílio), optamos pela aplicação semanal de 3 milhões de unidades de IFNa.

O procedimento iniciou-se em 14 de janeiro de 2014 e completou-se em 29 de abril de 2014 (houve atraso devido a episódios, após a quarta aplicação, de cefaleia inespecífica e alterações de comportamento que exigiram a interrupção provisória para realização de exames de imagens e investigação laboratorial e endocrinológica que não exibiram alterações significativas; os sintomas tiveram remissão espontânea durante o restante do tratamento). Em 29 de abril de 2014, realizou-se a última aplicação do IFNa: o aspecto do conteúdo do reservatório apresentavase translúcido, hialino. A paciente e seus familiares negavam quaisquer queixas - clínica, neurológica ou comportamental. 


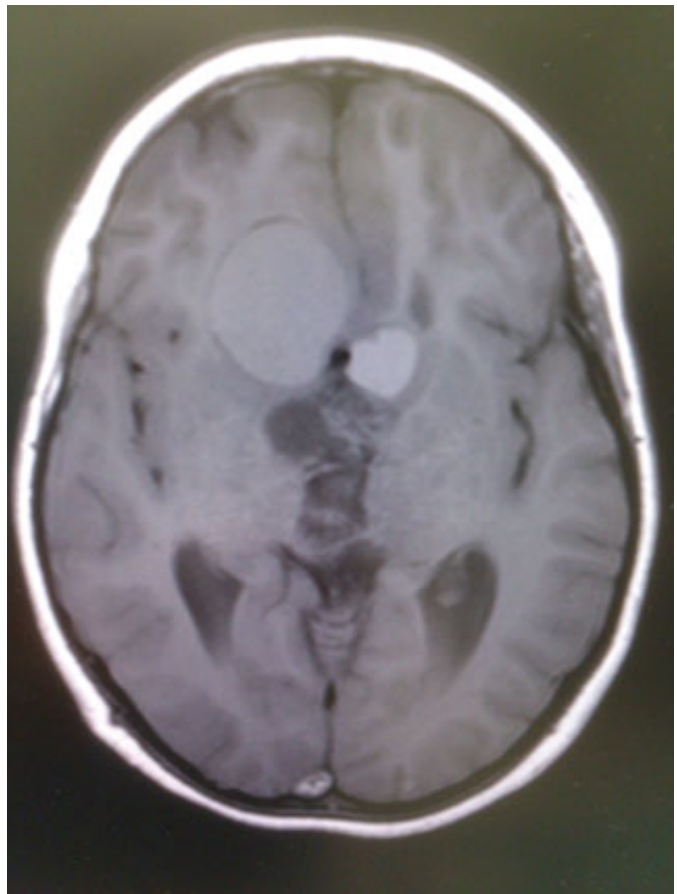

Fig. 1 Corte axial de RNM de crânio ponderada em T1, sem contraste, realizada na admissão no $\mathrm{HCB}$, evidenciando a lesão predominantemente cística bilateral.

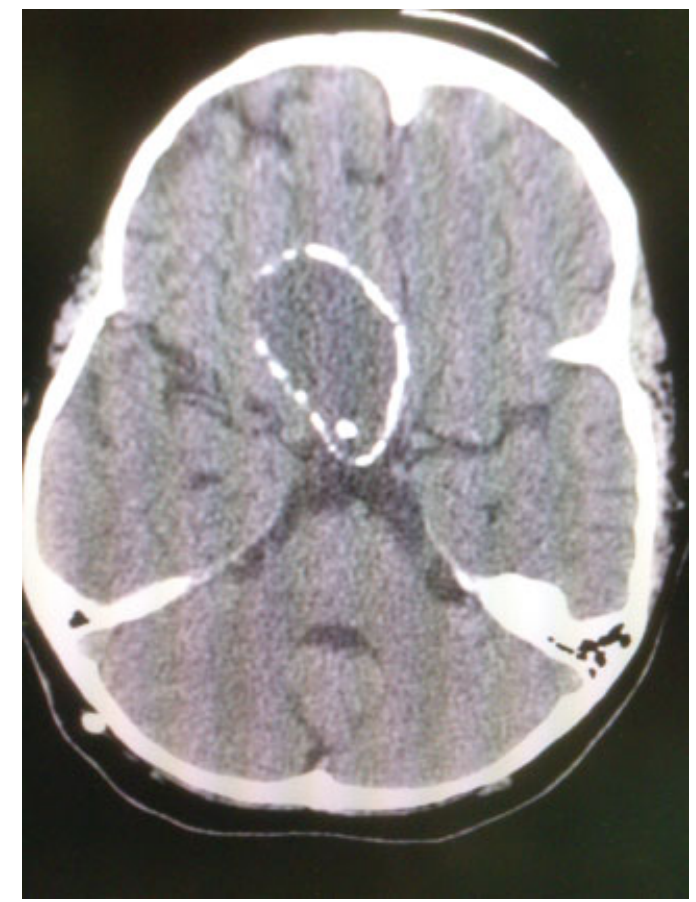

Fig. 2 Corte axial de TC de crânio de controle pós-operatório, evidenciando a localização adequada do cateter intracístico à direita.

Ressonância nuclear magnética de crânio de controle foi realizada em 12 de maio de 2014, evidenciando regressão importante do componente cístico localizado à direita, associado à ausência de hipersinal de seu conteúdo remanescente na sequência $\mathrm{T} 1$.

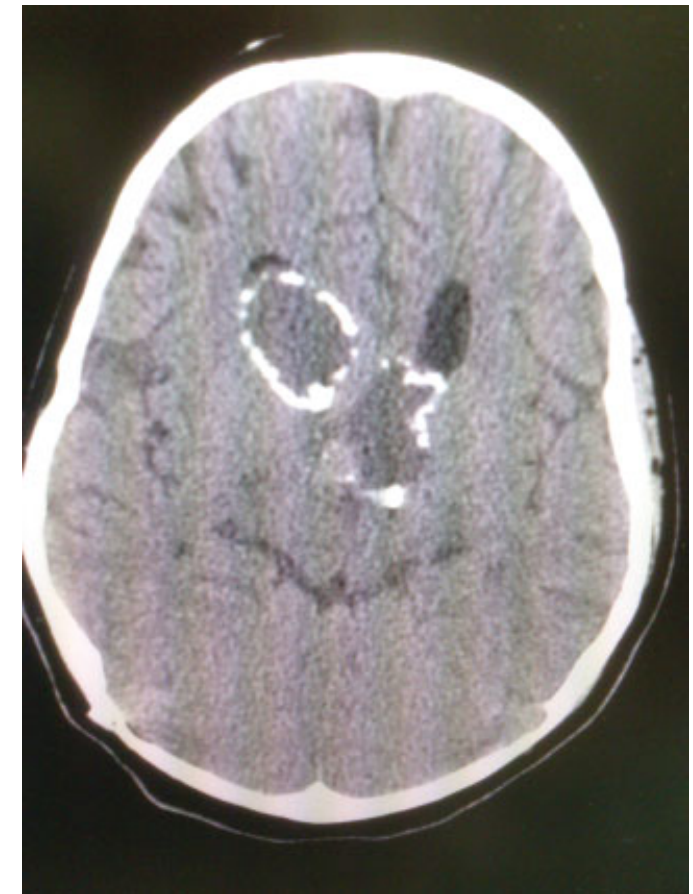

Fig. 3 Corte axial de TC de crânio de controle pós-operatório mostrando cateter intracístico mal locado à esquerda, ultrapassando a parede posterior deste.

\section{Discussão}

Em 2005, Cavalheiro et al. ${ }^{10}$ relataram pela primeira vez o uso bem sucedido de IFNa intracístico através de cateter em nove crianças portadoras de craniofaringiomas císticos. Em 2010, os autores apresentaram os resultados do tratamento com IFNa, de casuística multicêntrica, em sessenta pacientes. ${ }^{15}$ Todos os pacientes foram submetidos à inserção intracística de cateter ligado a um reservatório Ommaya subgaleal. Cinco dias depois, o posicionamento do cateter e a presença de fístula eram verificados pela injeção de contraste pelo reservatório; confirmado o posicionamento do cateter, realizava-se a aspiração tanto quanto possível do fluido cístico e a injeção de 3 milhões de unidades de IFNa no interior do cisto, através do reservatório. 0 procedimento se repetiu em dias alternados até completar doze injeções (36 milhões de unidades). Em alguns pacientes, foram utilizadas até três aplicações adicionais de IFNa, em caso de resposta insatisfatória, constatada em exames de imagens. Progressão ou recidiva do cisto, ou manutenção de sua intensidade de sinal em sequência ponderada em T1 de ressonância magnética, permitiria repetição das aplicações, decorridos 30 dias das aplicações iniciais. ${ }^{2}$ Os autores obtiveram controle do tumor (redução tumoral maior que $50 \%$ ) em 47 pacientes, o que corresponde a $78,3 \%$ dos casos. ${ }^{15}$ Ressecção cirúrgica foi necessária em 13 pacientes devido à progressão tumoral; 8 pacientes apresentaram novas disfunções endocrinológicas após o tratamento. Os seguintes sintomas adversos atribuídos ao IFNa - facilmente controlados e que, além de desaparecerem completamente depois do término do tratamento, não impediram a continuidade das aplicações 


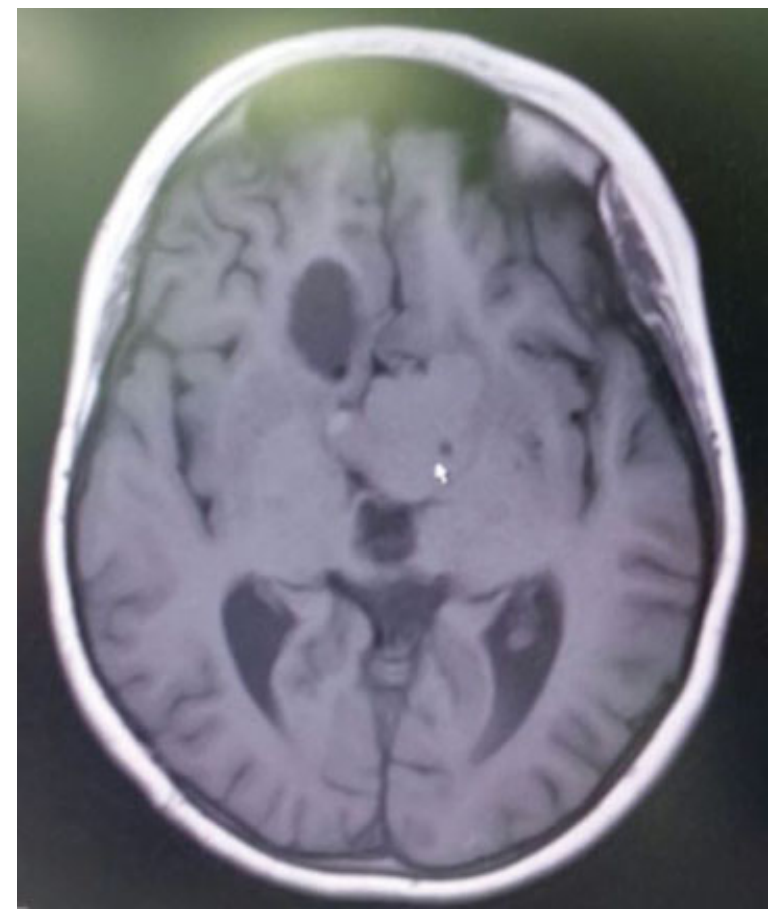

Fig. 4 Corte axial de RNM de crânio ponderada em T1, sem contraste, após o término do tratamento, constatando a regressão da lesão, a ausência de hipersinal em T1 no seu interior à direita, e o crescimento da lesão contralateral (seta).

- foram relatados por 18 pacientes (30\%): cefaleia, febre, edema palpebral e síndrome de fadiga crônica.

O protocolo de tratamento utilizado por Cavalheiro et al. ${ }^{15}$ foi adotado para tratamento de craniofaringioma cístico em crianças pelo Sick Children Hospital, em Toronto, Canadá. ${ }^{5}$ No tratamento de nossa paciente, também utilizamos esse protocolo, porém, com intervalos semanais em vez de dias alternados. Tendo como parâmetro de resposta ao tratamento a redução do tamanho do cisto maior que $50 \%$ em relação às medidas prévias da lesão, e a intensidade do sinal na RM ponderada em T1, classificamos a resposta terapêutica como satisfatória (-Fig. 4).

A definição da melhor terapia para os craniofaringiomas persiste como desafio, semelhantemente à definição da posologia ideal relacionada às formas alternativas de tratamento, que deve considerar, entre outras coisas, tolerância, efeitos adversos, eficácia no controle da doença e adesão dos pacientes.

\section{Conclusão}

O caso relatado apresentou bons resultados com a utilização empírica de intervalos semanais para a aplicação de IFNa.
Reitera, destarte, a efetividade da terapia intracística com IFNa para o controle dos craniofaringiomas predominantemente císticos. Entretanto, novos estudos são necessários para definir adequadamente o esquema posológico (dose apropriada e número de ciclos) desta quimioterapia intratumoral.

\section{Referências}

1 Buchfelder M, Schlaffer SM, Lin F, Kleindienst A. Surgery for craniopharyngioma. Pituitary 2013;16(1):18-25

2 Steinbok P, Hukin J. Intracystic treatments for craniopharyngioma. Neurosurg Focus 2010;28(4):E13

3 Müller HL. Childhood craniopharyngioma-current concepts in diagnosis, therapy and follow-up. Nat Rev Endocrinol 2010;6(11): 609-618

4 Müller HL. Childhood craniopharyngioma. Pituitary 2013;16(1): 56-67

5 Bartels U, Laperriere N, Bouffet E, Drake J. Intracystic therapies for cystic craniopharyngioma in childhood. Front Endocrinol (Lausanne) 2012;3:39

6 Pratheesh R, Swallow DM, Rajaratnam S, et al. Incidence, predictors and early post-operative course of diabetes insipidus in paediatric craniopharygioma: a comparison with adults. Childs Nerv Syst 2013;29(6):941-949

7 Barkhoudarian G, Laws ER. Craniopharyngioma: history. Pituitary 2013;16(1):1-8

8 Dastoli PA, Nicácio JM, Silva NS, et al. Cystic craniopharyngioma: intratumoral chemotherapy with alpha interferon. Arq Neuropsiquiatr 2011;69(1):50-55

9 Marchal JC, Klein O, Thouvenot P, Bernier V, Moret C, Chastagner P. Individualized treatment of craniopharyngioma in children: ways and means. Childs Nerv Syst 2005;21(8-9):655-659

10 Cavalheiro S, Dastoli PA, Silva NS, Toledo S, Lederman H, da Silva MC. Use of interferon alpha in intratumoral chemotherapy for cystic craniopharyngioma. Childs Nerv Syst 2005;21(8-9): 719-724

11 Cáceres A. Intracavitary therapeutic options in the management of cystic craniopharyngioma. Childs Nerv Syst 2005;21(8-9): 705-718

12 Rodríguez-Villanueva J, McDonnell TJ. Induction of apoptotic cell death in non-melanoma skin cancer by interferon-alpha. Int $\mathrm{J}$ Cancer 1995;61(1):110-114

13 Jakacki RI, Cohen BH, Jamison C, et al. Phase II evaluation of interferon-alpha-2a for progressive or recurrent craniopharyngiomas. J Neurosurg 2000;92(2):255-260

14 Cavalheiro S, Sparapani FV, Franco JO, da Silva MC, Braga FM. Use of bleomycin in intratumoral chemotherapy for cystic craniopharyngioma. Case report. J Neurosurg 1996;84(1):124-126

15 Cavalheiro S, Di Rocco C, Valenzuela S, et al. Craniopharyngiomas: intratumoral chemotherapy with interferon-alpha: a multicenter preliminary study with 60 cases. Neurosurg Focus 2010;28(4): E12 